\title{
The AAO's Gemini High-resolution Optical SpecTrograph (GHOST) concept
}

\author{
Michael J. Ireland ${ }^{a, f^{*}}$, Stuart Barnes ${ }^{b}$, David Cochrane ${ }^{c}$, Matthew Colless ${ }^{a}$, Peter Connor ${ }^{c}$, \\ Anthony Horton $^{a}$, Steve Gibson ${ }^{c}$, Jon Lawrence $^{a}$, Sarah Martell ${ }^{a}$, Peter McGregor ${ }^{d}$, Tom Nicolle $^{c}$, \\ Kathryn Nield ${ }^{c}$, David Orr ${ }^{a}$, J. Gordon Robertson ${ }^{a, e}$, Stuart Ryder ${ }^{a}$, Andrew Sheinis ${ }^{a}$, \\ Greg Smith ${ }^{a}$, Nick Staszak ${ }^{a}$, Julia Tims $^{a}$, Pascal Xavier ${ }^{a}$, Peter Young ${ }^{d}$ and Jessica Zheng ${ }^{a}$ \\ ${ }^{a}$ Australian Astronomical Observatory, PO Box 296, Epping, NSW 2121, Australia; \\ ${ }^{b}$ Stuart Barnes Optical Design, P.O. Box 157, Lyttelton 8841, New Zealand; \\ ${ }^{c}$ Industrial Research Ltd., 69 Gracefield Road, PO Box 31310, Lower Hutt 5040, New Zealand; \\ ${ }^{d}$ Research School of Astronomy \& Astrophysics, Cotter Rd., Weston Creek, ACT 2611, Australia; \\ ${ }^{e}$ Sydney Institute for Astronomy, School of Physics, University of Sydney, NSW 2006, Australia; \\ ${ }^{f}$ Department of Physics and Astronomy, Macquarie University, NSW 2109, Australia
}

\begin{abstract}
The Gemini High-Resolution Optical SpecTrograph (GHOST) will fill an important gap in the current suite of Gemini instruments. We will describe the Australian Astronomical Observatory (AAO)-led concept for GHOST, which consists of a multi-object, compact, high-efficiency, fixed-format, fiber-fed design. The spectrograph itself is a four-arm variant of the asymmetric white-pupil echelle Kiwispec spectrograph, Kiwisped, produced by Industrial Research Ltd. This spectrograph has an R4 grating and a 100mm pupil, and separate cross-disperser and camera optics for each of the four arms, carefully optimized for their respective wavelength ranges. We feed this spectrograph with a miniature lensletbased IFU that sub-samples the seeing disk of a single object into 7 hexagonal sub-images, reformatting this into a slit with a second set of double microlenses at the spectrograph entrance with relatively little loss due to focal-ratio degradation. This reformatting enables high spectral resolution from a compact design that fits well within the relatively tight GHOST budget. We will describe our baseline 2-object R 50,000 design with full wavelength coverage from the ultraviolet to the silicon cutoff, as well as the high-resolution single-object $\mathrm{R} \sim 75,000$ mode.
\end{abstract}

Keywords: spectrograph, spectroscopy, echelle, high resolution, radial velocity

\section{INTRODUCTION}

The Gemini consortium have prioritized the development of a new high-resolution optical spectrograph for the Gemini telescope(s). Gemini's design strength for seeing-limited infrared and target of opportunity observations is a single Cassegrain focus, with several instruments that can be easily switched via a tertiary fold mirror. This feature is problematic for high-resolution spectroscopy, however, because a large instrument has to either maintain adequate stability over a wide range of gravity vectors, or be placed a distance of tens of meters from the focus via a fiber cable. In late 2011, the Gemini Observatory awarded three competitive conceptual design studies for the Gemini HighResolution Optical Spectrograph (GHOS) instrument. The Australian Astronomical Observatory (AAO) in partnership with the Australian National University (ANU) conducted one of these studies. Our concept, opting for the more easily pronounced "Gemini High-Resolution Optical SpecTrograph" (GHOST), is based on a fiber-fed design incorporating a Kiwispec spectrograph. In this paper, we will describe an overview of the GHOST instrument concept and a more detailed description of the image-plane reformatting optics.

\subsection{Science Context}

As a workhorse instrument, GHOST is expected to meet a wide range of science goals. Gemini provided 14 science cases derived from earlier White Papers. Most of these involved measuring abundances in different astrophysical contexts, 2 required spectropolarimetry and only one required a long slit. Desired wavelength ranges included the $310 \mathrm{~nm}$ to $1000 \mathrm{~nm}$ range, and only 3 of the 14 cases listed resolutions above 60,000 as being desirable. After consulting the Australian community, we added 3 additional science cases:

\footnotetext{
*mireland@aao.gov.au
}

Ground-based and Airborne Instrumentation for Astronomy IV, edited by lan S. McLean, Suzanne K. Ramsay, Hideki Takami, Proc. of SPIE Vol. 8446, 844629 - (c) 2012 SPIE · CCC code: 0277-786X/12/\$18 · doi: 10.1117/12.925746 
1. Exoplanet detection via radial velocity, particularly follow-up of fainter transiting exoplanet hosts, and exoplanet searches around $\mathrm{M}$ dwarfs. This science required no better than $2 \mathrm{~m} / \mathrm{s}$ calibration precision due to the relatively faint magnitudes of the targets, but did require a high efficiency spectrograph with broad wavelength coverage.

2. The study of time variation of fundamental constants through redshifted quasar absorption systems. Previous work in this area has been limited by the long-term calibration precision of other spectrographs on $8 \mathrm{~m}$ class telescopes. This science case required at least $10 \mathrm{~m} / \mathrm{s}$ long-term radial velocity precision.

3. Absorption spectroscopy of Gamma-Ray Bursts. Of greatest interest are both the properties of the GRB host galaxies, and how the gas in the galaxies at high redshift is modified by the very strong radiation field from the explosion. These observations require the Rapid Target of Opportunity (RToO) mode of Gemini, benefit from the widest simultaneous wavelength range possible and require a resolution of 40,000 or greater.

The science requirements resulting from these science cases are given in Table 1.

Table 1. The science requirements for GHOST.

\begin{tabular}{|l|l|}
\hline Title & Requirement \\
\hline Wavelength range & GHOST shall provide simultaneous wavelength coverage from 363nm to 1000nm \\
\hline Spectral resolution & $\begin{array}{l}\text { GHOST shall have two selectable spectral resolution modes: standard resolution mode with R>50,000 } \\
\text { and high resolution mode with R }>75,000 .\end{array}$ \\
\hline Sensitivity & $\begin{array}{l}\text { GHOST shall obtain a sensitivity of m=18.0 in a 1 hour observation for 30 sigma per resolution element } \\
\text { in standard resolution mode in dark time (50th sky brightness percentile) at a wavelength of 500 nm. }\end{array}$ \\
\hline Targets and field size & $\begin{array}{l}\text { GHOST shall have the capability to observe 2 targets simultaneously over a 7.5 arcmin diameter field of } \\
\text { view. }\end{array}$ \\
\hline $\begin{array}{l}\text { Radial velocity } \\
\text { precision }\end{array}$ & $\begin{array}{l}\text { GHOST shall provide a radial velocity precision of 200 } \mathrm{m} / \mathrm{s} \text { over the full wavelength range in standard } \\
\text { resolution mode and shall have the capability to provide a radial velocity precision of 2 m/s over the full } \\
\text { wavelength range for the high spectral resolution mode. }\end{array}$ \\
\hline Spatial Sampling & GHOST shall spatially sample each target object over a field size of 1.2 arcsec. \\
\hline Spectro-polarimetry & GHOST should provide a spectropolarimetric capability that can distinguish all Stokes parameters. \\
\hline
\end{tabular}

\section{INSTRUMENT CONCEPT OVERVIEW}

The GHOST instrument concept comprises multi-object integral field units (IFUs) with individual Atmospheric Dispersion Compensators (ADCs) that are positionable at the Cassegrain focal plane, feeding a fiber bundle that transports light to a gravity-invariant thermally-controlled spectrograph mounted on the rotating carousel. Key design features for GHOST are as follows:

- Broad Simultaneous Wavelength Coverage. A simultaneous wavelength coverage of 363 to $1000 \mathrm{~nm}$. The short end of this wavelength cutoff met all science goals other than $\mathrm{N}$ and $\mathrm{O}$ abundances in cool, metal poor stars, where the $\mathrm{NH}$ and $\mathrm{OH}$ bands lie, and a key Be doublet at $313 \mathrm{~nm}$ are located.

- Focal-plane image-slicing. GHOST finely samples the stellar PSF at the focal plane over a 1.2" diameter field of view with 7 elements (standard-resolution mode) or 19 elements (high-resolution mode) and reformats this into a spectrograph slit. This gives a very large saving in spectrograph cost, weight and throughput compared to alternative approaches.

- Microlens-based IFUs. GHOST uses a pair of microlens arrays at the focal plane to inject light into the fibers. This approach gives added versatility in spectrograph and input optics design, and has highefficiency ( $>90 \%$ of a fiber-only design).

- Miniature-ADCs. A dedicated Atmospheric Dispersion Compensator (ADC) is provided for each IFU. This approach allows the ADCs to comprise small optics giving significant improvements in blue throughput.

- Dual slits. Two separate slits are fed from IFUs with a different scale of image sampling. This provides two separate spectral resolution modes: "standard" and "high". 
- Multi-object positioning system. Two Commercial Off The Shelf (COTS) xy stages allow positioning of two IFUs within the Cassegrain field of view, providing a substantial gain in observing efficiency.

- Broadspectrum optical fibers. Broad bandwidth fibers with good blue throughput and focal ratio degradation properties are used for the fiber bundle. Measured throughputs of more than $80 \%$ were achieved for the $20.5 \mathrm{~m}$ fiber length applicable to the carousel location at all wavelengths (or $70 \%$ for a possible pier location).

- Asymmetric white-pupil echelle spectrograph. The spectrograph is a variant on the Kiwispec $100 \mathrm{~mm}$ pupil spectrograph with an R4 grating which is a relatively compact and tested design using a single catalog echelle grating.

- Four-arm spectrograph design. The spectrograph has 4 arms with a common collimator and main dispersing element. This design maximizes throughput, enables thermo-electric cooling of relatively small $(2 \mathrm{~K} \times 2 \mathrm{~K})$ detectors in 2 of the 4 arms, and provides wide simultaneous wavelength coverage.

- Volume-phase holographic (VPH) grism cross-dispersers. The use of VPH grisms for the spectrograph cross-dispersion results in a relatively high throughput, especially given the relatively narrow wavelength range of each arm.

- Slit-viewing camera. A dedicated slit-viewing camera views a $1 \%$ reflection of the spectrograph input and a $100 \%$ output from a series of guide fibers. This obviates the necessity for an on-instrument wavefront sensor, and provides an exposure meter and spectral Point-Spread Function (PSF) monitor.

- Thermally controlled chamber. The spectrograph is enclosed inside a thermally-controlled chamber. This provides high spectral stability and maintenance/adjustment-free operation.

- High radial velocity precision mode. Simultaneous wavelength calibration, pressure calibration, and fiber agitation provide a very high precision radial velocity mode, with only minor impact to cost and complexity. This mode is based on the CYCLOPS2 upgrade to the UCLES spectrograph on the Anglo-Australian Telescope.

- Spectropolarimetry mode. A fixed module is proposed as an upscope option that provides polarimetric information.

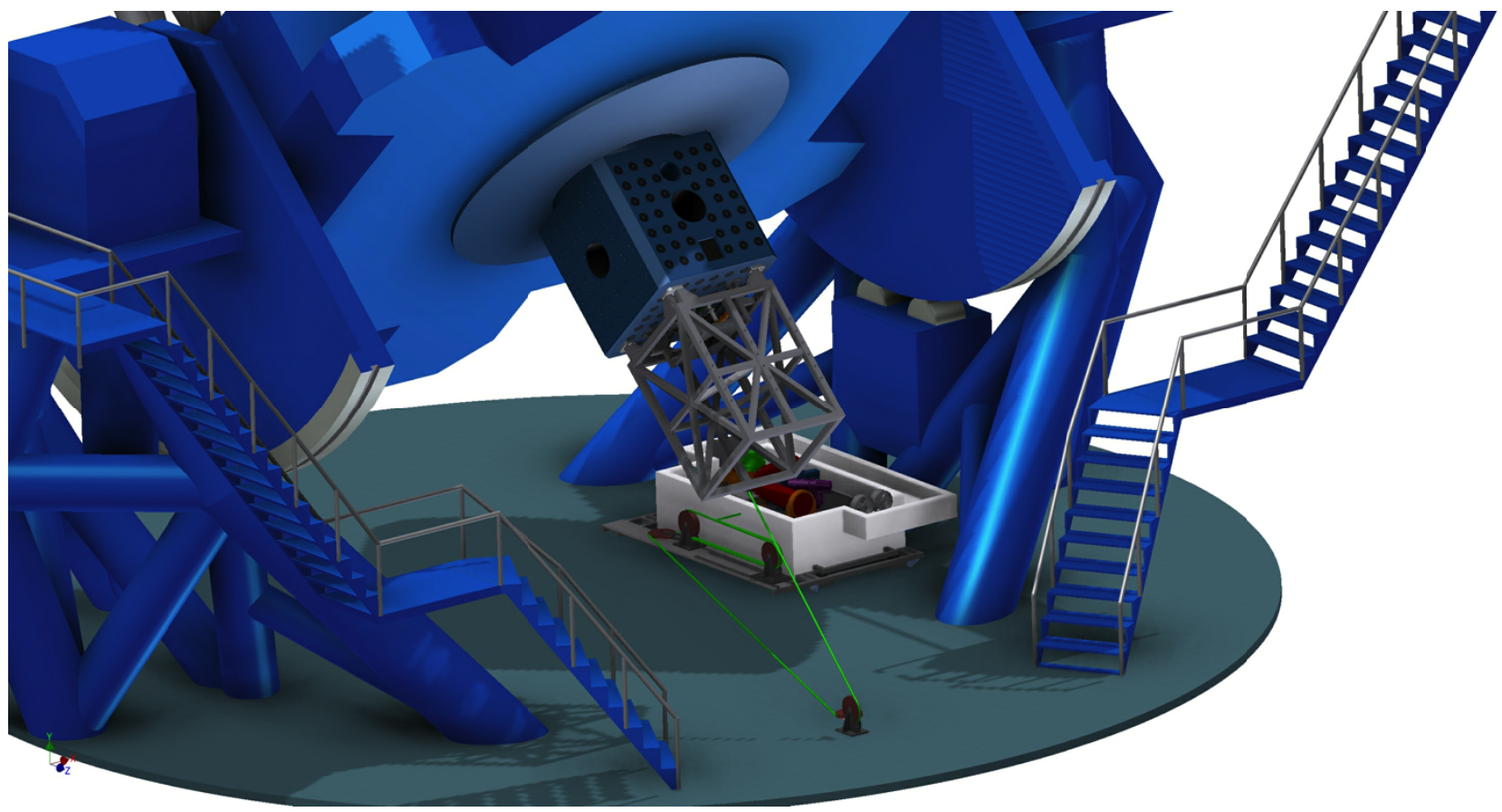

Figure 1. The AAO-GHOST spectrograph in its preferred location on the carousel, showing the fiber cable when the telescope is 45 degrees from zenith. The spectrograph is shown in its thermal enclosure but with its top surfaces removed. 


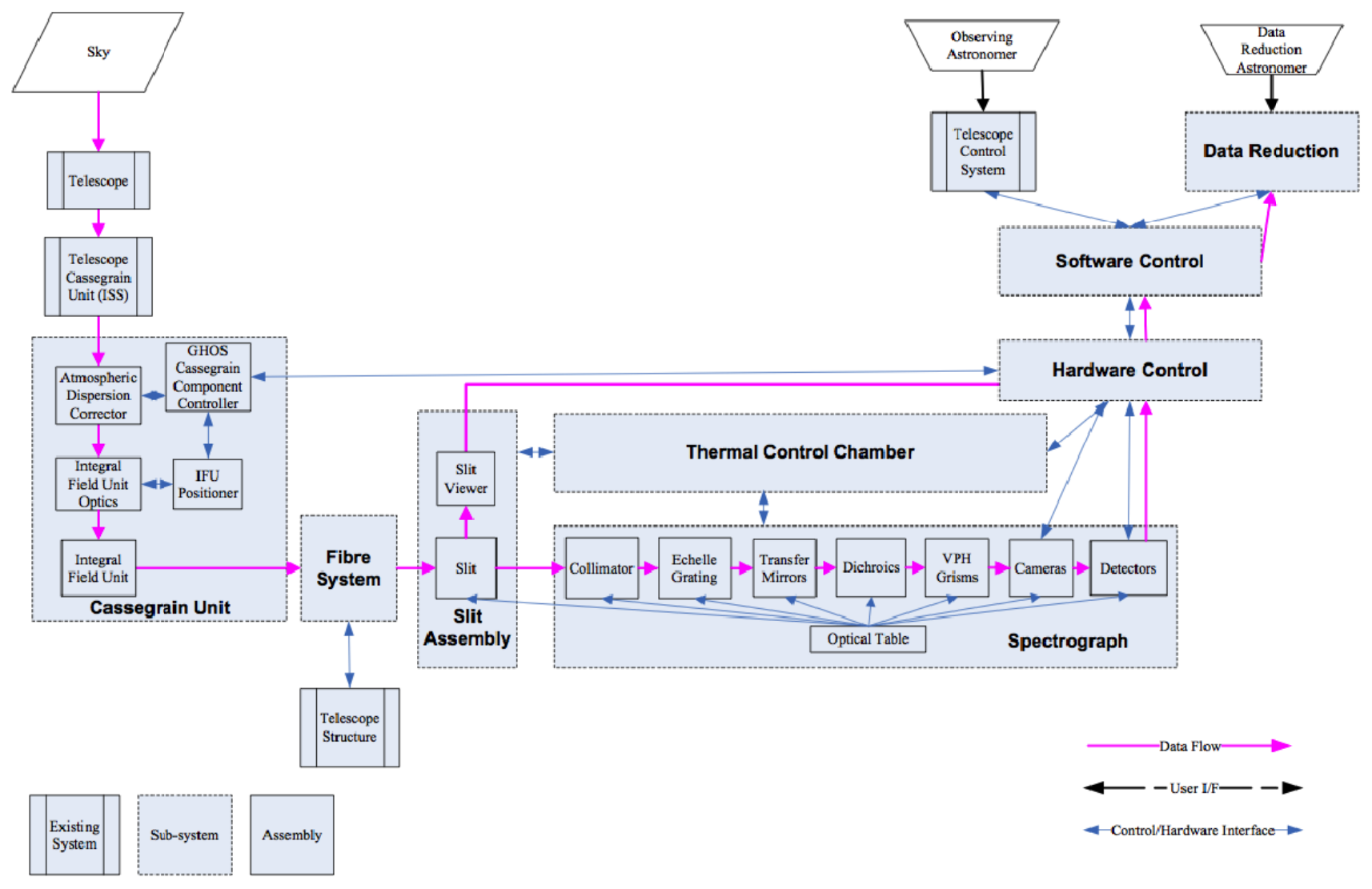

Figure 2. A system-level overview of the AAO-GHOST concept. The spectrograph subsystem is a Kiwispec variant, produced by IRL, the hardware and software control is to be completed by ANU, and the remaining subsystems are the responsibility of the AAO.

Figure 1 shows the spectrograph and Cassegrain unit in location at Gemini, and Figure 2 gives a system level overview of the AAO-GHOST concept, and The light path in the system diagram goes from the telescope to the Cassegrain unit, where two positioners each with their individual ADCs enable positioning over a full 7.5 arcminute field of view. Starlight then travels through a fiber-cable to a reformatted slit and then to the Kiwispec spectrograph. Further details on each element follow.

The Cassegrain unit forms a pupil image on the fiber face of each Integral Field Unit (IFU) element for each positioner. This requires a telecentricity correction lens, an atmospheric dispersion corrector for each IFU, and two micolenses for each IFU element. The use of a double-microlens is described further in Section 3. In addition, a large ballast weight (structure shown in Figure 1) is required to maintain Gemini's center of gravity requirements - this is mechanically isolated from the mounts for the optical elements. Figure 3 shows the main opto-mechanical elements of the Cassegrain unit, and Figure 4 shows the Atmospheric Dispersion Correctors (ADCs) and IFU mount. The ADCs are capable of placing the full 363-1000nm spectral range well within a single 0.4" IFU element with an internal 
transmission exceeding 97\%, as shown in Figure 5. Figure 6 depicts a mock-up of the user interface for controlling the positioners.

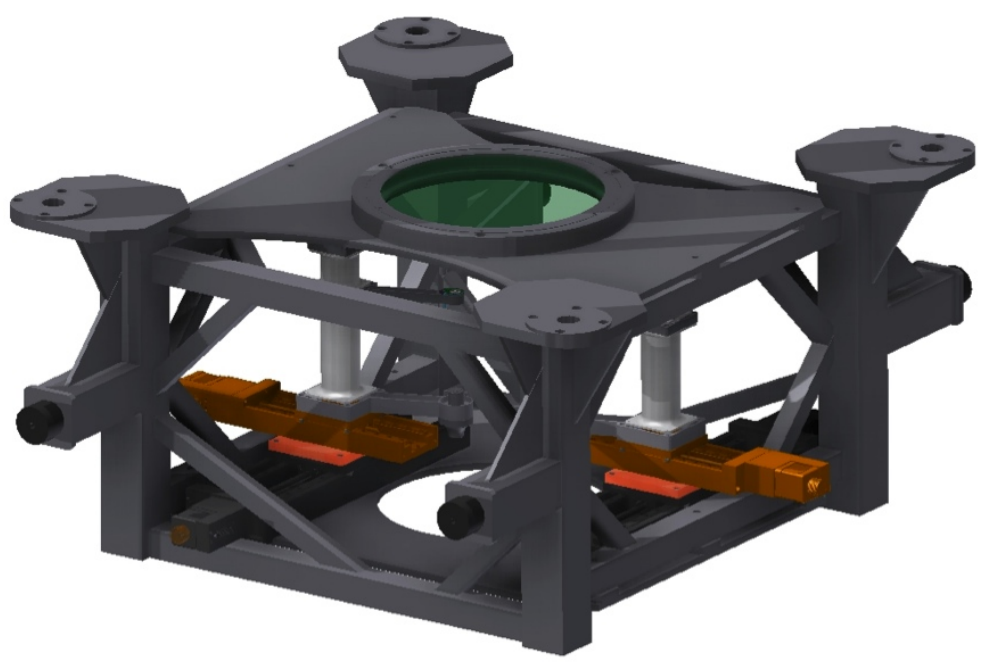

Figure 3. The Cassegrain unit, excluding the ballast weight. The orange and black elements are the COTS stages from Physiks Instrumente, that position the two IFUs over a 7.5 arcminute field of view. The first (green) element is a telecentricity correction lens, which ensures that the pupil image formed on the fiber faces is centered irrespective of where the object is in the field of view.
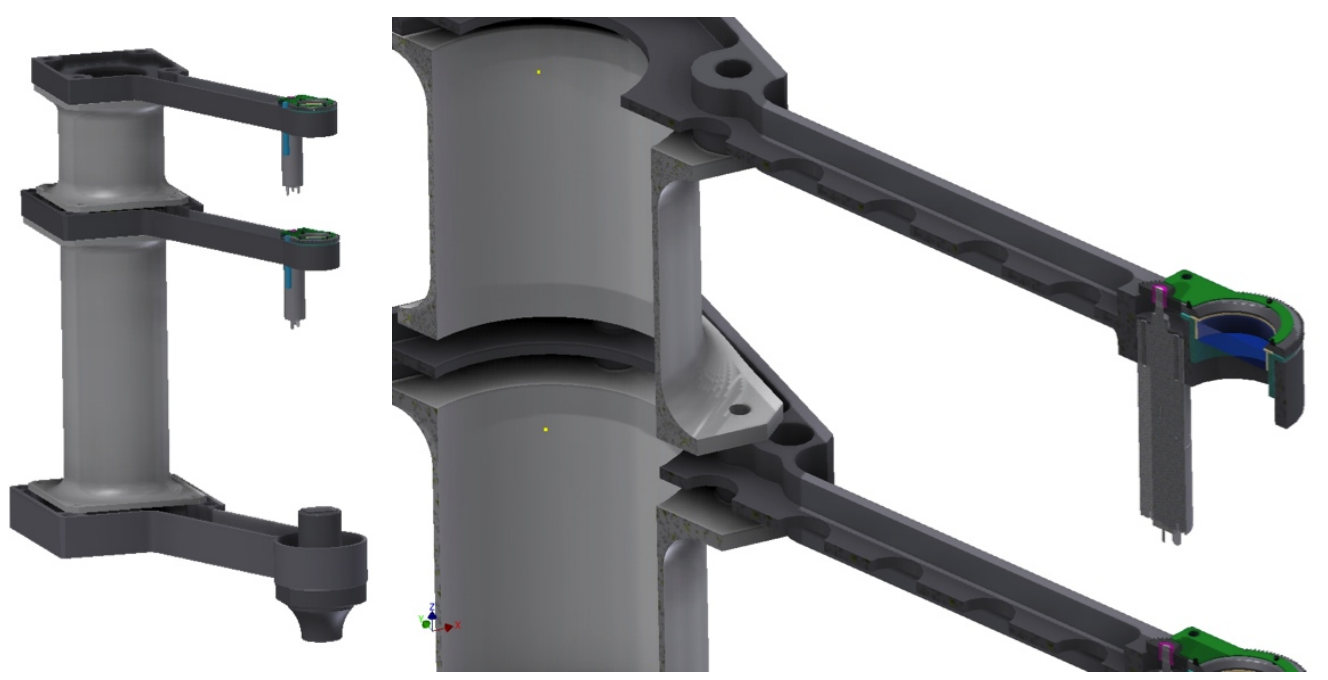

Figure 4. A close-up of the ADCs for one IFU. Each ADC consists of two rotating prism pairs with maximum clear aperture of $20 \mathrm{~mm}$. 


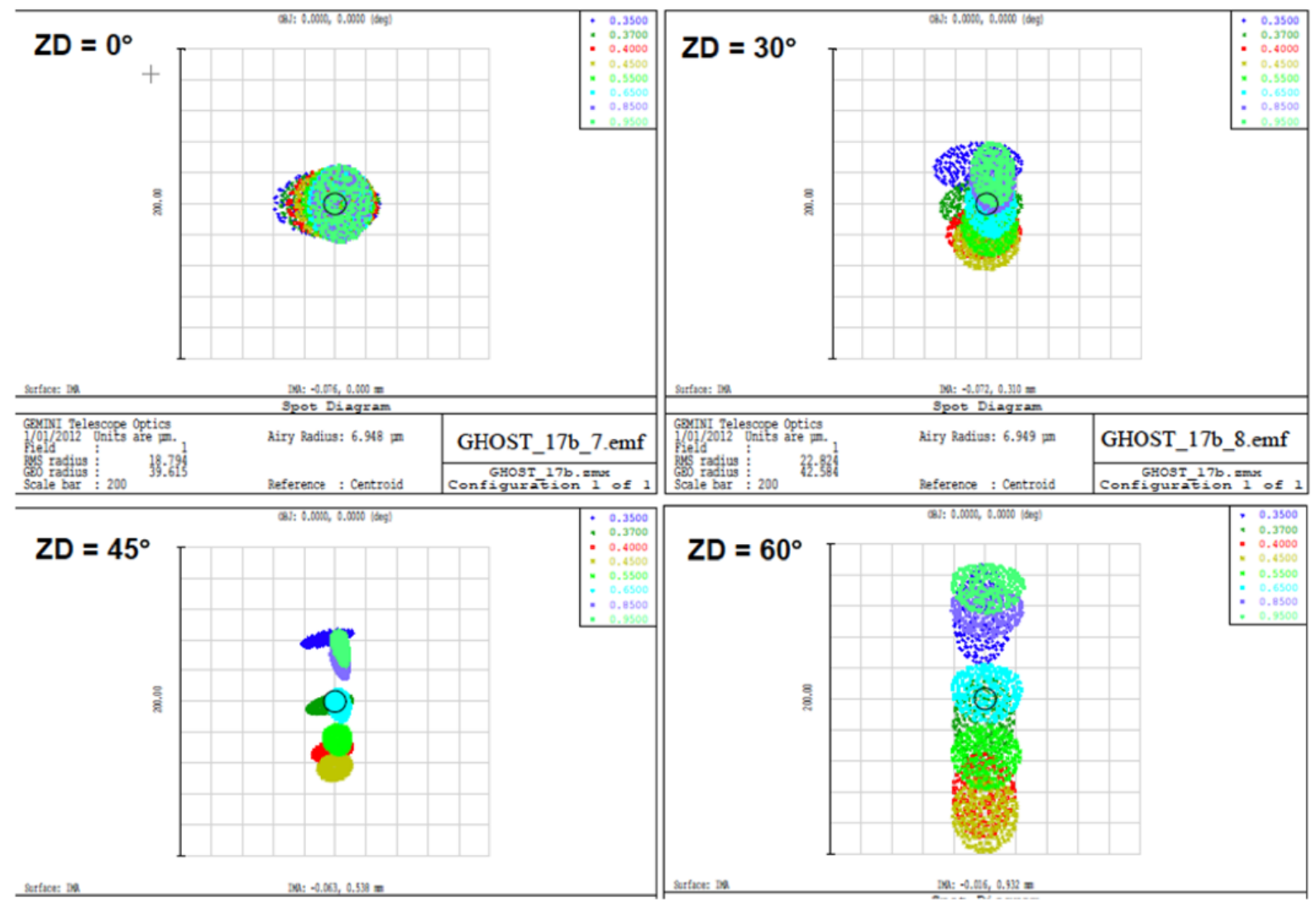

Figure 5. Spot diagrams for the ADC system, showing wavelengths between 350 and 950nm, from 0 to 60 degrees from zenith. The boxes are 200 microns on a side, which compares favorably to the 250 micron flat to flat standard resolution IFU element size.

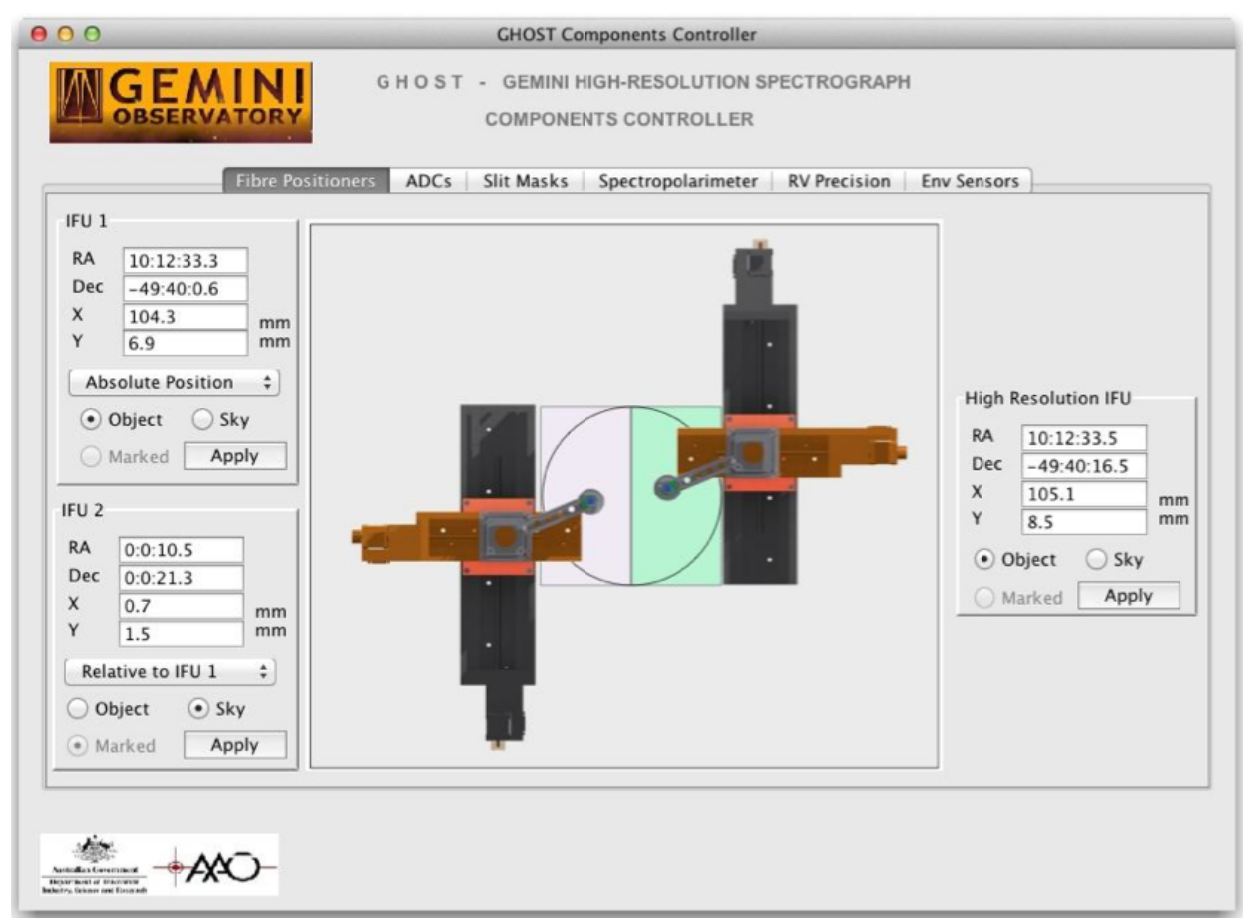

Figure 6. Mock-up of the GHOST components controller screen, showing the two IFU arms and their patrol fields which overlap on the centerline. Although the high-resolution IFU is physically part of IFU1, the user sees it as its own element. 
The spectrograph itself is an asymmetric white-pupil design as depicted in Figures 7 and 8, with the key parameters of the design given in Table 2 and image quality for the blue camera as an example given in Figure 9. The primary disperser, collimator and transfer collimator are closely based on the prototype Kiwispec ${ }^{1,2}$ instrument which was commissioned at Mt John Observatory, New Zealand in 2011. This instrument along with reduced on-sky data is described in Gibson et al ${ }^{1}$. The cross-dispersers are Volume-Phase Holographic (VPH) grisms, which are made of silica, and are designed to produce anamorphism in order to shrink the spectra in the cross-dispersion direction and be more efficient in the use of detector area. The pupil size in the primary dispersion direction (vertical) is $33 \mathrm{~mm}$, increasing up to $62 \mathrm{~mm}$ in the cross-dispersion direction. All reflective coatings other than the grating, collimator and first fold mirror are high efficiency dielectric coatings. The detector housing and control electronics are thermoelectrically cooled $850 \mathrm{~S}$ (blue and UV) or cryo-tiger cooled 1100S (green and red) cameras from Spectral Instruments. A final decision on CCDs to be used will be made at a later stage, in order to most carefully optimize quantum efficiencies in the limited wavelength ranges.

In addition to the standard resolution slit, the high-resolution slit is relayed to a position immediately above the standard resolution slit (see section 3). The only moveable parts in the spectrograph are a mask in front of the slit itself, and focus adjustments on the cameras. As the entire spectrograph is placed in a thermally controlled enclosure, the focus is only expected to be adjusted after the spectrograph is first temperature stabilized. A $1 \%$ reflection from an inclined glass plate enables both standard and high resolution slits to be imaged by a slit-viewing camera (lower left of Figure 7). Alignment fibers are also relayed to this camera, which has 3 purposes: an exposure meter, an alignment and flexure compensation system (feeding back to the positioners) and a spectral PSF monitor.

Design efficiencies for the spectrograph itself excluding the CCD exceed $40 \%$ at order centers over the full 363 $1000 \mathrm{~nm}$ wavelength range (see Figure 12), using measured echelle efficiencies and manufacturer curves for the VPH designs. Excluding slit losses, overall efficiency at $500 \mathrm{~nm}$ is required to exceed $26 \%$, with a goal of $39 \%$. Figures 11 and 12 show the raw data from a data simulator, illustrating the spectral format, and the close-packing of the 7 output IFU elements so that individual fiber outputs cannot be clearly distinguished. Finally, Figure 13 depicts the predicted signalto-noise as a function of Vega magnitude.

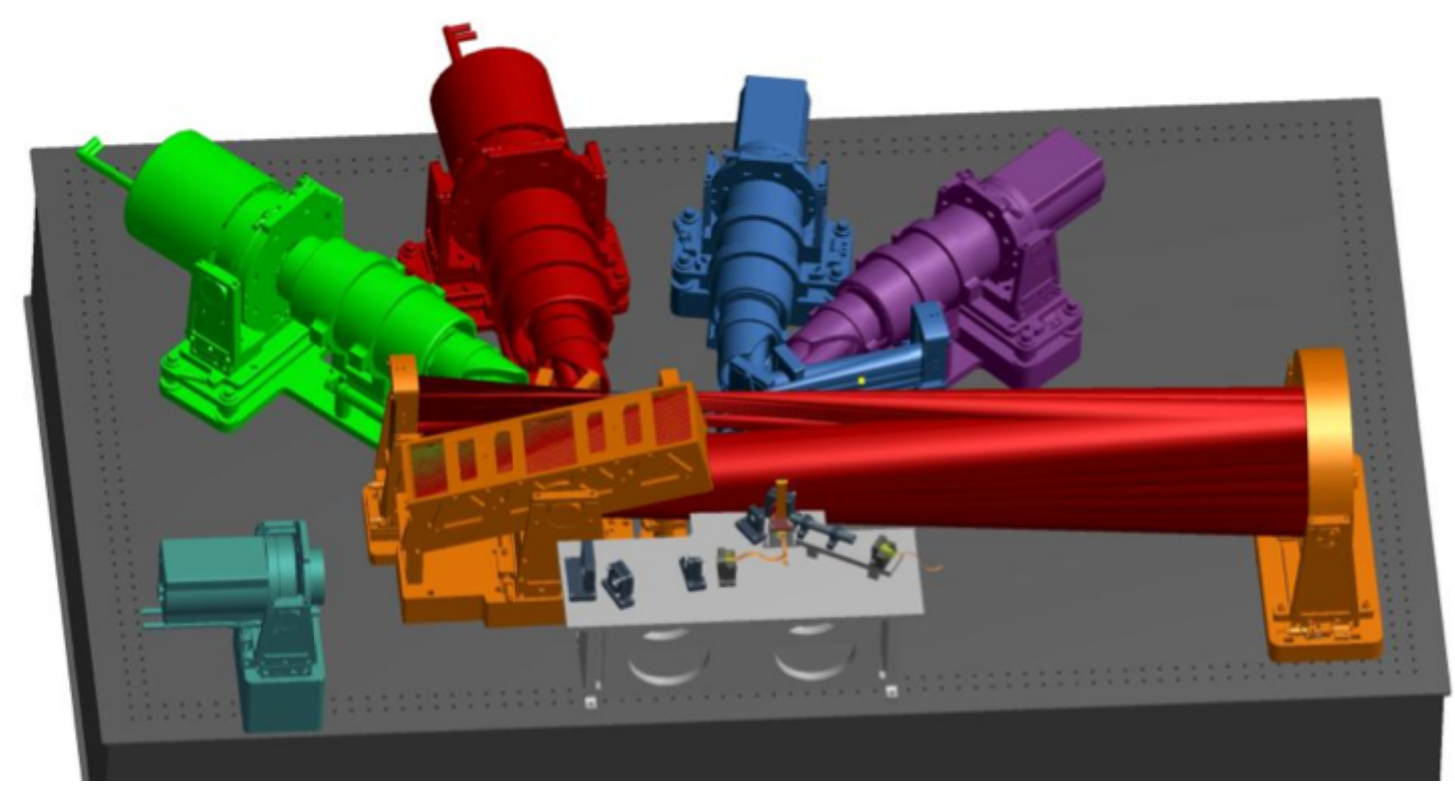

Figure 7. The opto-mechanical concept for the spectrograph, showing the common optical elements to all 4 arms (orange) as well as the Green, Red, Blue and UV (purple) arms. The slit assembly optics are on their own small optical table in the lower part of the figure, both for rigidity and to ease the integration between the AAO and Kiwistar elements. 


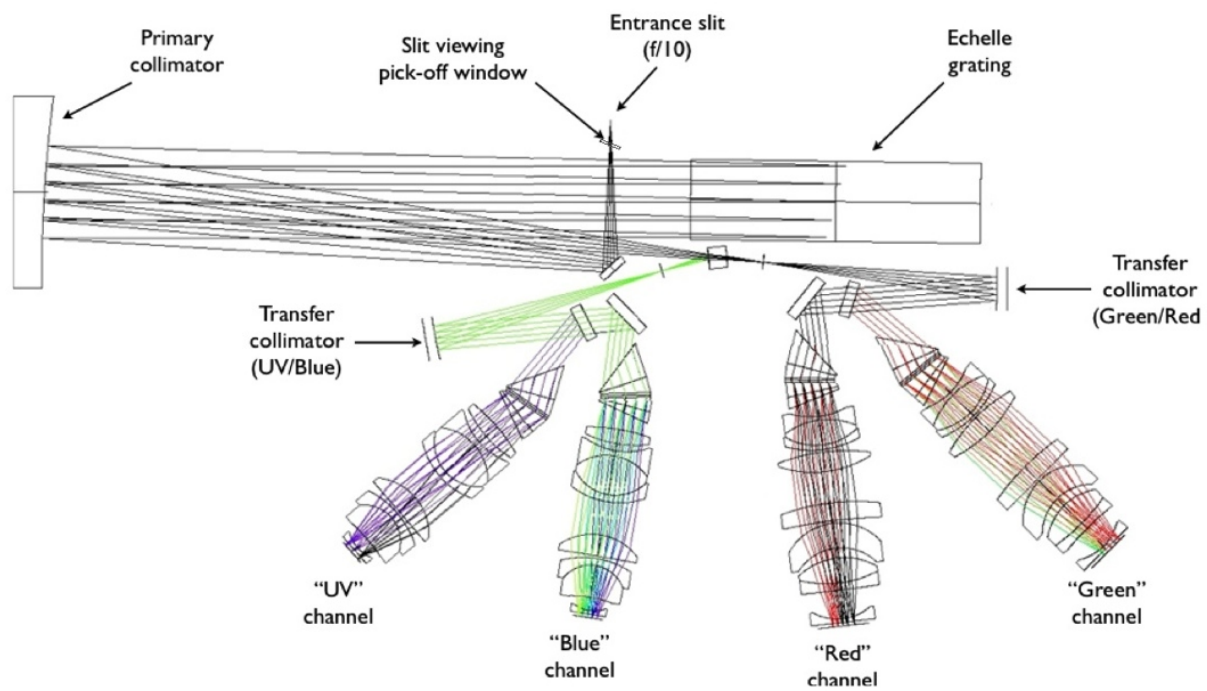

Figure 8. Optical layout of the spectrograph, showing the key common elements. All dichroics in the system are long-pass, with intermediate slit planes after the first dichroic that enable additional baffling to avoid scattered light. The grisms perform both crossdispersion and anamorphism, to shrink the spectra in the cross-dispersion direction.

Table 2. Summary of the Kiwispec-GHOST properties.

\begin{tabular}{|c|c|c|c|c|}
\hline \multirow{2}{*}{$\begin{array}{l}\text { Property } \\
\text { Name: }\end{array}$} & \multicolumn{4}{|c|}{ Value } \\
\hline & UV & Blue & Green & Red \\
\hline $\begin{array}{l}\text { Echelle grating: } \\
\text { Blaze angle }\left(\theta_{\mathrm{B}}\right): \\
\text { Line density }\left(T_{\text {ech }}\right):\end{array}$ & & $\begin{array}{r}75.135^{\circ} \\
31.6 \mathrm{li}\end{array}$ & $\begin{array}{l}\text { (nominal) } \\
\text { les/mm }\end{array}$ & \\
\hline $\begin{array}{l}\text { Beam size(s): } \\
\text { Collimated: } \\
\text { White pupil: }\end{array}$ & & $33.3 \mathrm{~mm}$ (ignori & $\begin{array}{l}\mathrm{mm} \\
\mathrm{gg} \text { anamorphism) }\end{array}$ & \\
\hline $\begin{array}{l}\text { Orders }(m): \\
\text { Range (max-min): } \\
\text { Number: }\end{array}$ & $\begin{array}{c}169-139 \\
31\end{array}$ & $\begin{array}{c}140-110 \\
31\end{array}$ & $\begin{array}{c}111-83 \\
29\end{array}$ & $\begin{array}{c}84-61 \\
24\end{array}$ \\
\hline $\begin{array}{l}\text { Wavelengths }(\mathrm{nm}) \text { : } \\
\text { Min, max: } \\
\text { FSR overlap }{ }^{\mathrm{a}}:\end{array}$ & $\begin{array}{c}362<\lambda_{\mathrm{B}}<440 \\
1.51-1.82\end{array}$ & $\begin{array}{c}437<\lambda_{\mathrm{B}}<556 \\
1.18-1.49\end{array}$ & $\begin{array}{c}551<\lambda_{\mathrm{B}}<737 \\
1.82-2.43\end{array}$ & $\begin{array}{c}728<\lambda_{\mathrm{B}}<1003 \\
1.31-1.80\end{array}$ \\
\hline $\begin{array}{l}\text { Cross disperser }{ }^{\mathrm{b}}: \\
\quad \theta_{\mathrm{i}}: \\
T_{\text {xdp }}: \\
\theta_{\mathrm{f}}:\end{array}$ & $\begin{array}{c}60.00^{\circ} \\
2575 \text { lines } / \mathrm{mm} \\
15.45^{\circ}\end{array}$ & $\begin{array}{c}61.00^{\circ} \\
1750 \text { lines } / \mathrm{mm} \\
15.67^{\circ}\end{array}$ & $\begin{array}{c}61.00^{\circ} \\
1150 \text { lines } / \mathrm{mm} \\
15.35^{\circ}\end{array}$ & $\begin{array}{c}55.00^{\circ} \\
775 \text { lines } / \mathrm{mm} \\
15.85^{\circ}\end{array}$ \\
\hline $\begin{array}{l}\text { Anamorphic factors }{ }^{\mathrm{c}}: \\
A_{\text {ech }}: \\
A_{\mathrm{xdp}}:\end{array}$ & $\begin{array}{l}0.88-1.11 \\
1.77-1.89\end{array}$ & $\begin{array}{l}0.86-1.12 \\
1.84-1.88\end{array}$ & $\begin{array}{l}0.81-1.16 \\
1.72-1.74\end{array}$ & $\begin{array}{l}0.72-1.22 \\
1.54-1.58\end{array}$ \\
\hline $\begin{array}{l}\text { Camera focal length: } \\
\text { Detector: } \\
\text { Pixel number }\left(n_{\mathrm{x}} \times n_{\mathrm{y}}\right) \text { : } \\
\text { Pixel size: }\end{array}$ & $\begin{array}{c}2048 \times 2048 \\
13.5 \mu \mathrm{m}\end{array}$ & $\begin{array}{c}2048 \times 2048 \\
13.5 \mu \mathrm{m}\end{array}$ & $\begin{array}{c}4096 \times 2048 \\
15.0 \mu \mathrm{m}\end{array}$ & $\begin{array}{c}4096 \times 2048 \\
15.0 \mu \mathrm{m}\end{array}$ \\
\hline $\begin{array}{r}\text { a. Defined as } \Delta \lambda_{\mathrm{CCD}} / \Delta \lambda_{\mathrm{FS}} \\
\Delta \lambda_{\mathrm{FSR}}=\text { wavelength e } \\
\Delta \lambda_{\mathrm{CCD}}=\text { wavelength } \\
\text { b. Where the following defi } \\
\theta_{\mathrm{i}}=\text { angle of incidence } \\
T_{\mathrm{xdp}}=\mathrm{VPH} \text { line density, } \\
\theta_{\mathrm{f}}=\text { angle of exitance } \\
\text { c. The anamorphic factors } \\
A_{\text {ech }}=B_{\mathrm{x}}^{\prime} / B_{\mathrm{x}} \text { (due to } \\
A_{\mathrm{xdp}}=B_{\mathrm{y}}^{\prime} / B_{\mathrm{y}} \text { (due to } \\
B \text { and } B^{\prime} \text { are the collin }\end{array}$ & $\begin{array}{l}\text { where: } \\
\text { tent of one free sp } \\
\text { tent of the detect } \\
\text { itions apply: } \\
\text { irst grism surface, } \\
\text { st grism surface. } \\
\text { e defined as follow } \\
\text { chelle dispersion), } \\
\text { /PH grism cross-di } \\
\text { ated beam width/1 }\end{array}$ & $\begin{array}{l}\text { ctra range, and } \\
\text { r. } \\
\text { s: } \\
\text { spersion). }\end{array}$ & & \\
\hline
\end{tabular}



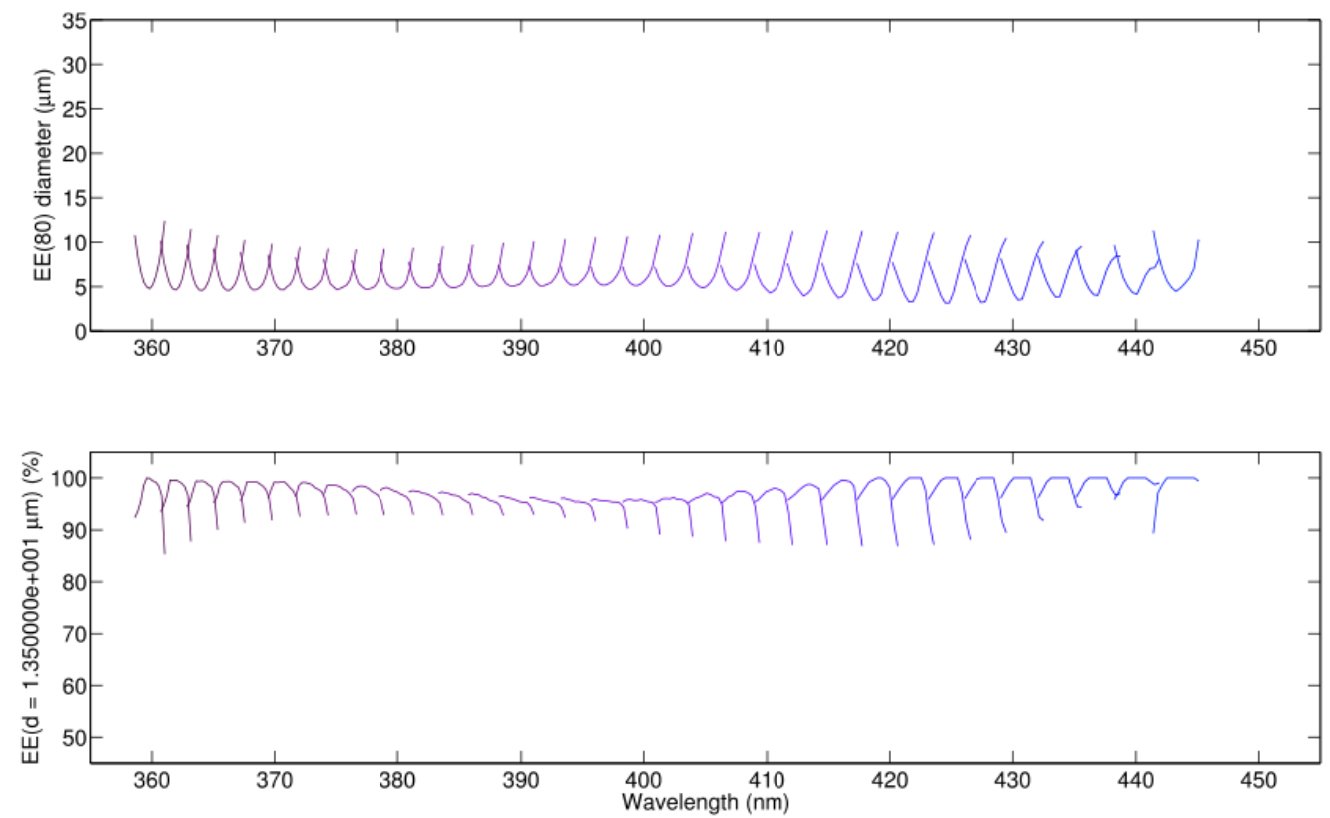

Figure 9. Image quality of the KiwiSpec-GHOST UV channel. The diameter at EE(80) is shown in the top panel, whereas the bottom panel shows the encircled energy within a $13.5 \mu \mathrm{m}$ diameter circle (the side length of one pixel).

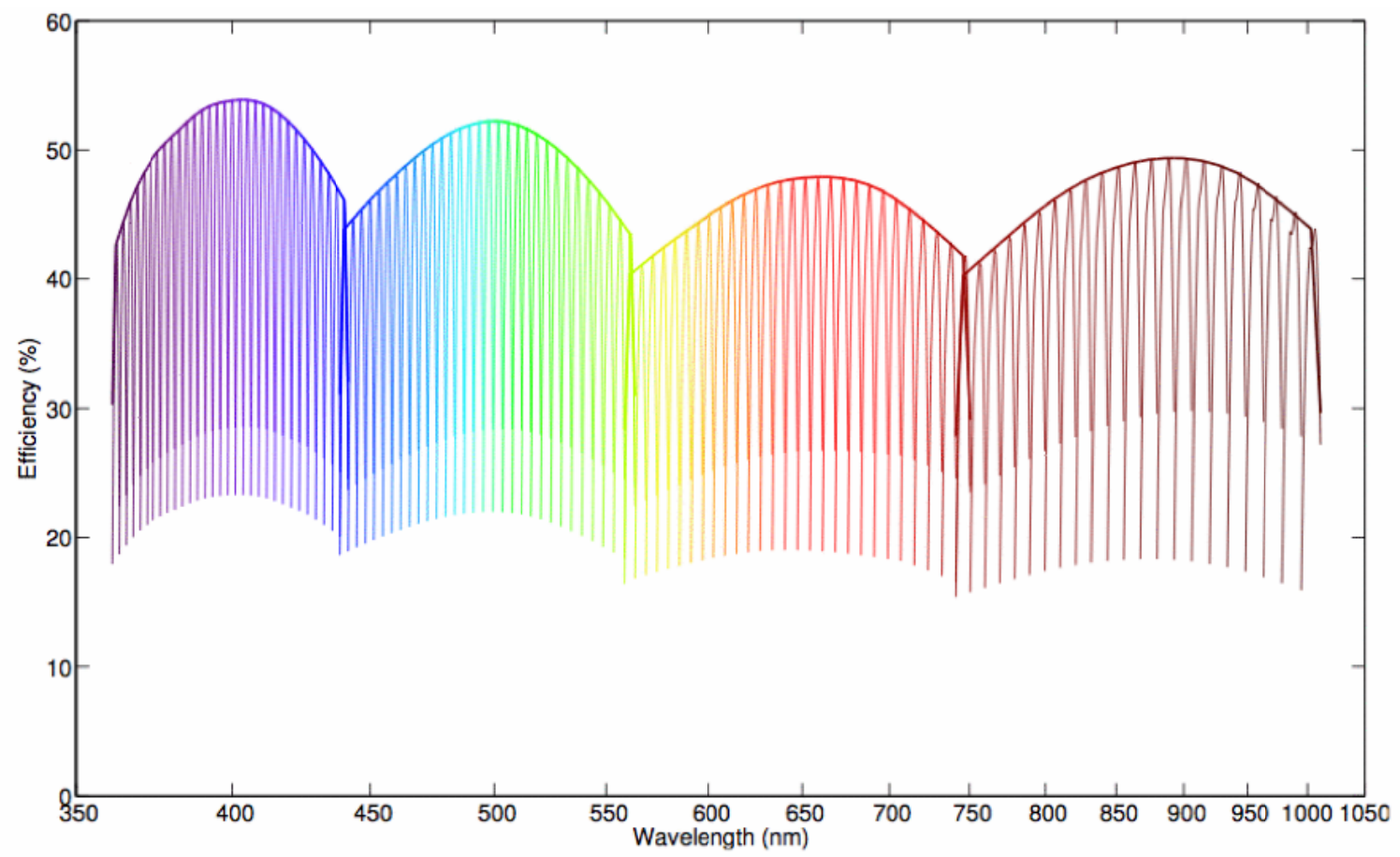

Figure 10. Design efficiencies for the 4-arm Kiwispec, from the slit to the detector, not including the CCD quantum efficiency, and not including the effects of the dichroics (which enable one at least one order to be sampled in two detectors at each cutoff). More than one free spectral range is available in all orders (see Table 2), so the edges of each order are sampled twice. 


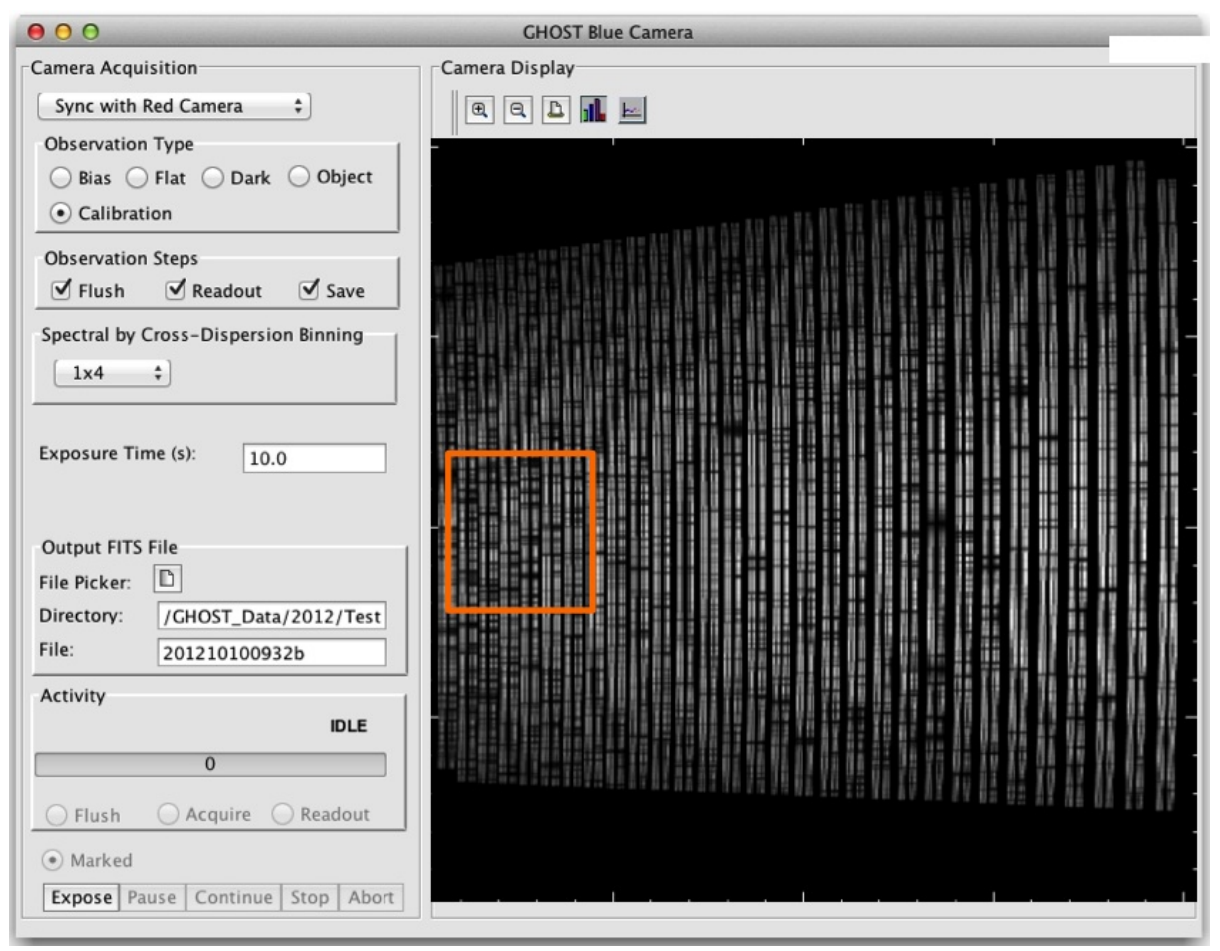

Figure 11. An example engineering GUI screen-shot for the Blue camera, for simulated data where two objects are observed in the standard resolution mode. Only one free-spectral range is shown, and the simulations include order curvature.

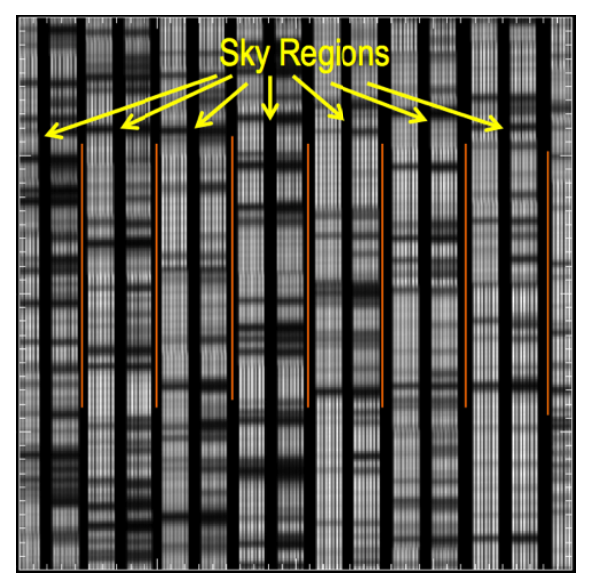

Figure 12. A zoom in on the orange boxed region of Figure 7, showing the gaps between orders (orange) and the regions between each object which can be used for sky subtraction when observing two objects. When observing only one object, the second IFU will be positioned on sky, with beam switching possible in order to obtain the best possible sky subtraction. 


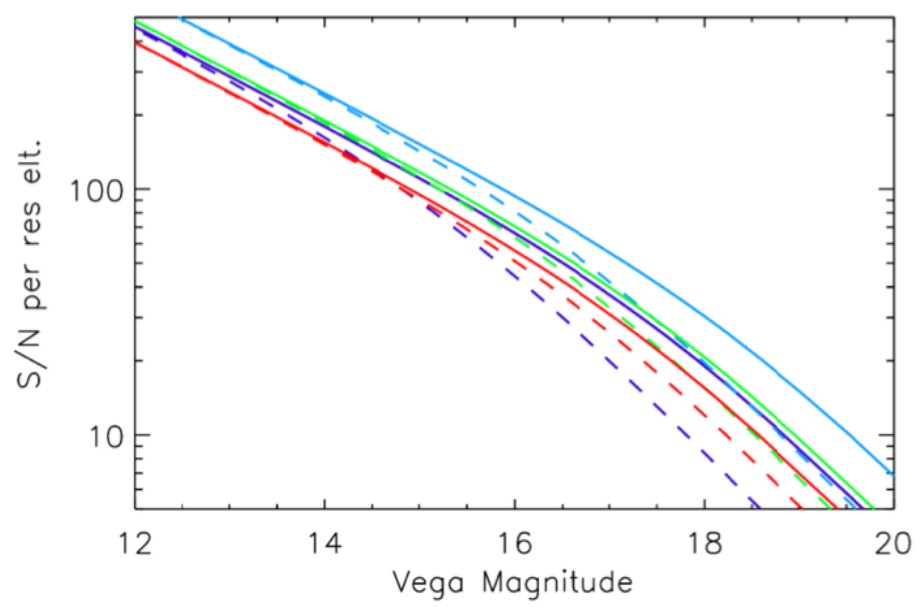

Figure 13. Signal-to-noise as a function of Vega magnitude for a 1 hour exposure including overheads in dark time (solid lines) and bright time (dashed lines), for order centers at $400 \mathrm{~nm}$ (violet), $500 \mathrm{~nm}$ (blue), 650nm (green) and $850 \mathrm{~nm}$ (red), based on minimum required performance specifications and a conservative sky subtraction approach. The signal-to-noise between sky lines for the 650 and $850 \mathrm{~nm}$ wavelengths will in practice be greater than this figure, which uses an average sky brightness over R and I bands.

\section{IMAGE-PLANE REFORMATTING}

The most unique part of the AAO-GHOST concept is arguably the multi-object capability without sacrificing wavelength coverage, and the use of microlenses to reformat the image-plane. Figures 16 and 17 illustrate what this reformatting looks like in the input image-plane and output slit plane, while Figure 18 illustrates how the double microlens configuration images the input pupil onto the fiber input face so that the image of the hexagonal microlens (the nominal telescope focal plane) is placed at infinity. This arrangement minimizes geometrical focal-ratio degradation. Injecting a fiber with a single microlens would give measurable focal-ratio degradation whenever the ratio of lenslet diameter to fiber core size is smaller than approximately 50 . We have prototyped the two-microlens arrangement, with a 19 element fiber array provided by Fiberguide Industries. Both microlenses are diffraction limited within our initial testing uncertainties $(\sim 0.5$ waves at $633 \mathrm{~nm})$ with scattering at a $<1 \%$ level. The most critical alignment is between microlens 2 and the fiber array, which is at the $<1$ micron level.

On output, the double-microlens arrangement places images of the fiber faces at infinity. This means that the collimator then forms overlapping images of all fiber faces on the echelle grating, and the transfer collimator again forms images of the fiber faces on the VPH grating. This arrangement means that there are no efficiency losses due to FRD only losses in spectral resolution. In fact, if spectral resolution is measured in terms of FWHM, we do not see any decrease in spectral resolution in our models or tests due to FRD - only an increase in the wings of the spectral PSF, making it closer to Gaussian. Contrary to early papers on $\mathrm{FRD}^{1}$, we have measured smaller FRD effects from our smallest core fibers ( 35 micron core diameter for the high-resolution mode) than more traditional $\sim 100$ micron core fibers $^{2}$. 

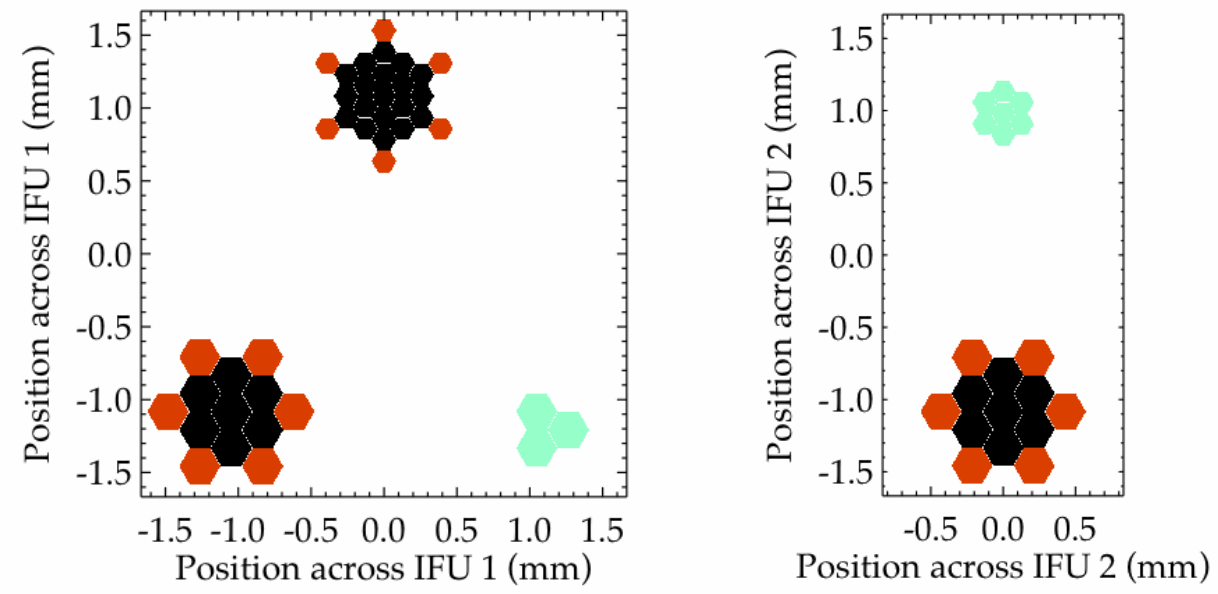

Figure 14. The arrangement of the input hexagonal micro-lenses in the image-plane, for the two IFUs. The larger black hexagons are the standard resolution $(\mathrm{R}=50 \mathrm{~K})$ IFU elements, and the smaller black hexagons are the high resolution $(\mathrm{R} \sim 75 \mathrm{~K})$ microlens elements. The green IFU elements are allocated to sky. The orange/red IFU elements are fibers used for acquisition and flexure compensation, with $100 \%$ of their received flux viewed by the slit-viewing camera.

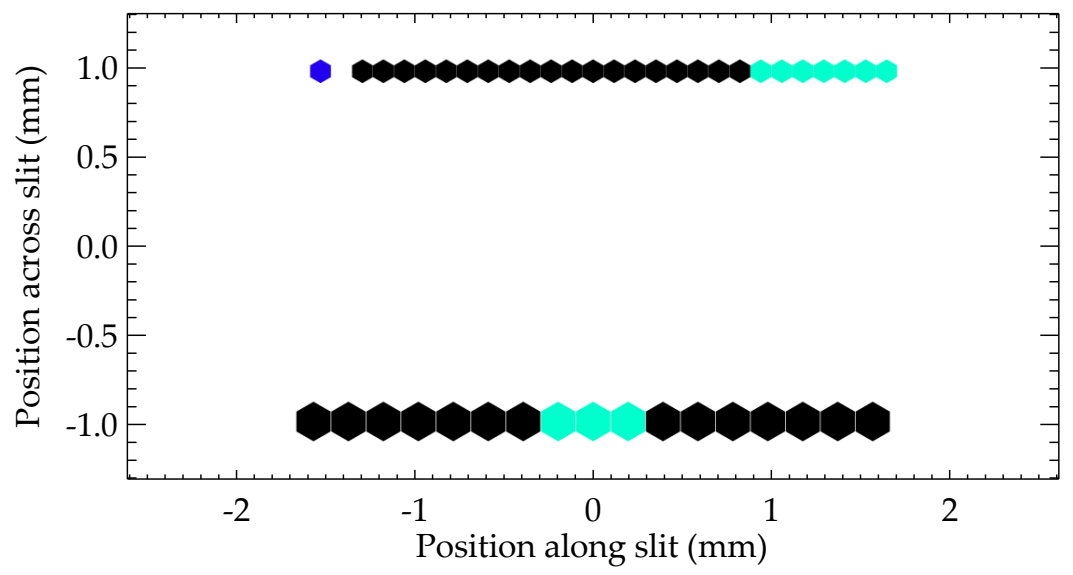

Figure 15. The arrangement of the output micro-lenses in the slit plane, showing the standard resolution (lower) and high resolution (upper) slits, with science (black) and sky (green) IFU elements. Only one slit is illuminated at a time. The blue IFU element in the high resolution slit is a simultaneous $\mathrm{Th} / \mathrm{Ar}$ reference fiber. 


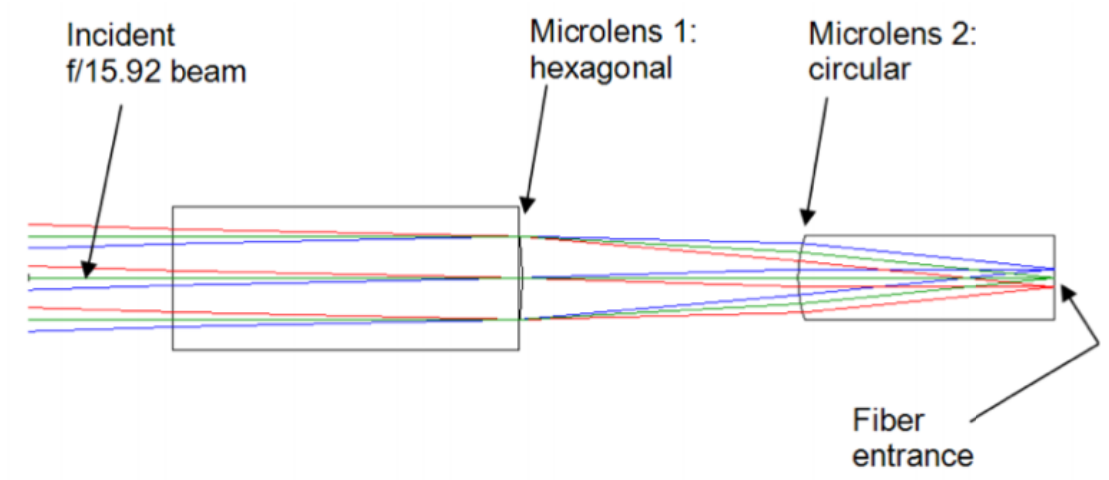

Figure 16. The double-microlens fiber injection optics. The first microlens is hexagonal with high (>97\%) fill factor, and the second microlens is a circular microlens on a hexagonal grid. This arrangement is needed to avoid geometrical FRD: each point on the fiber entrance is illuminated by the same cone. There are similar double-microlens arrangements on output, and for both standard resolution and high-resolution modes. On output, the microlens assembly accepts a 20\% smaller f-ratio to allow for FRD.

\section{CONCLUSIONS}

The AAO's concept for the Gemini High-Resolution Optical SpecTrograph (GHOST) provides a low-risk, versatile design for the Gemini telescope that falls within the target budget. Reformatting using microlens arrays enables a 2object $\mathrm{R} \sim 50,000$ or 1 -object $\mathrm{R} \sim 75,000$ spectrograph to be constructed with only a $100 \mathrm{~mm}$ pupil and a single COTS echelle grating. The 2-object standard resolution mode in particular uses either COTS components or components that have already been successfully prototyped during the conceptual design phase. The spectrograph itself is a variant of the Kiwispec spectrograph that has already been commissioned. The asymmetric white-pupil design grating gives a $33 \mathrm{~mm}$ pupil on entrance to the cross-dispersers and cameras, resulting in a significant cost and complexity saving. Should this concept be selected, commissioning will be anticipated in the first half of 2015.

\section{REFERENCES}

1. S. Gibson, S.I. Barnes, K. Nield, D. Cochrane, J. Hearnshaw, "KiwiSpec - an advanced spectrograph for high resolution spectroscopy: Prototype design and performance" in Ground-based and Airborne Instrumentation for Astronomy IV, Proc. of the SPIE, 2012 (submitted)

2. S.I. Barnes, S. Gibson, K. Nield, D. Cochrane, "KiwiSpec - an advanced spectrograph for high resolution spectroscopy: Optical design and variations" in Ground-based and Airborne Instrumentation for Astronomy IV, Proc. of the SPIE, 2012 (submitted)

3. L.W. Ramsey, "Focal ratio degradation in optical fibers of astronomical interest" in Fiber optics in astronomy, ASP conference series, 3, 26, 1988

4. D.M. Haynes, M.J. Withford, J.M Dawes, J.S. Lawrence, R. Raynes, Relative contributions of scattering, diffraction and model diffusion to focal ratio degradation in optical fibres, Mon. Not. R. Astron. Soc, 414, 253, 2011 